\title{
汽轮机调节阀和调节级特性建模与配汽优化
}

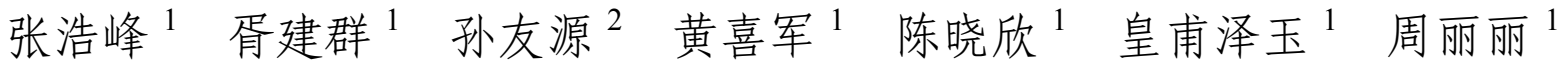 \\ (1. 东南大学能源热转换及其过程测控教育部重点实验室 南京 210096;
}

\author{
2. 华电电力科学研究院有限公司 杭州 310030)
}

\begin{abstract}
摘要: 针对喷嘴配汽汽轮机, 对调节阀和调节级联合进行建模, 通过计算得到调节阀和调节级组合特性曲线, 建立理论计算 模型。该模型便于工程应用, 利用调节阀和调节级组合的压比与划门开度可计算得到准确的调节阀流量。基于计算模型, 以 某 $300 \mathrm{MW}$ 机组为研究对象, 实现调节阀配汽函数的优化, 并得到机组顺序阀流量特性; 联合负荷和阀位, 对其配汽方式进 行优化。结果表明, 经过验证调节阀和调节级的组合特性模型能够准确地反映现场调节阀特性。根据该模型实现流量特性的 优化, 得到合理的重叠度, 提高阀门可控性; 经过优化分析得到机组的最优滑压曲线, 采用优化后的配汽方式运行, 降低机 组低负荷运行时的热耗，提高机组的灵活性和调峰能力。该优化方法对现场试验寻优提供理论基础。
\end{abstract}

关键词: 变工况; 调节阀; 调节级; 组合流量特性; 配汽优化

中图分类号: TK261

\section{Characteristic Model of Combined Governing Valve and Governing Stage and Optimization of Steam Distribution of Steam Turbine}

\author{
ZHANG Haofeng $^{1} \quad$ XU Jianqun $^{1} \quad$ SUN Youyuan $^{2}$ HUANG Xijun ${ }^{1}$ \\ CHEN Xiaoxin ${ }^{1}$ HUANGFU Zeyu ${ }^{1}$ ZHOU Lili ${ }^{1}$
}

(1. Key Laboratory of Energy Thermal Conversion and Control of Ministry of Education,

Southeast University, Nanjing 210096;

2. Huadian Electric Power Research Institute, Hangzhou 310030)

\begin{abstract}
For the nozzle steam turbine, the combined flow characteristics calculation model of governing valve and governing stage is established by calculation, and tested with open literature experimental data. The accurate flow of the governing valve can be calculated with the model by using the combination pressure ratio and the valve opening, which is convenient for engineering application. Method a calculation model for the off-design performance of a $300 \mathrm{MW}$ steam turbine unit based on the combined valve model, and the valve distribution function is optimized, and the flow character of the sequential valve is obtained. Then the steam distribution optimization is analyzed by using the load and valve position. Analysis shows that the combined flow characteristics calculation model of governing valve and governing stage can reflect the characteristics of the valve accurately. According to the model, the flow characteristic is optimized, the reasonable overlap degree is obtained. The optimal slip pressure curve of the unit is obtained, which reduce the heat consumption during low-load operation of the unit and improves the flexibility and peak-regulating capacity of the unit and provides the theoretical basis for optimization test.
\end{abstract}

Key words: varying condition; governing valve; governing stage; the combination flow characteristics; optimization of steam distribution

\section{0 前言}

近年来我国电力发展面临新的形势, 随着国家 全面实施燃煤机组超低排放与节能改造, 促进推广 清洁发电技术等政策的开展, 煤电机组面临环保和

20190127 收到初稿, 20190615 收到修改稿
经济双重挑战 ${ }^{[1-3]}$ 。经济方面, 由于用电结构变化导 致电网峰谷差越来越大。此外, 可再生能源机组的 发电份额不断提升, 这不仅压缩了煤电机组发电比 重, 其间歇不稳定的发电特点还对电网的频率产生 冲击, 造成煤电机组的调峰任务加剧。环保方面, 煤电机组要保证运行指标达到国家排放限值和能耗 水平。在这样的环境下, 机组不得不提高深度调峰 和快速变负荷能力, 这使得机组在低负荷工况下运 
行, 经济性和安全性降低。因此, 优化调整该类型 机组配汽方式进行, 对提高机组灵活性, 提高效率 和节能降耗具有重要的意义。

主蒸汽流量是汽轮机运行的重要参数, 其准确 性影响机组运行的出力和热耗, 是机组运行优化的 前提。为减少压力损失, 通常不设置主蒸汽流量测 点, 主蒸汽流量无法直接测得。若采用给水流量或 凝结水流量测量值确定主蒸汽流量, 即使机组在稳 态运行时, 因机组控制回路闭环, 会导致测量值上 下波动引起主蒸汽流量周期性变化; 利用调节级级 后压力, 采用弗留格尔公式进行主蒸汽流量计算只 适合一定范围, 超出适用范围会产生较大的误差。

现代机组采用数字电液控制系统 (Digital electric hydraulic control system, DEH)控制阀门开度 实现主蒸汽流量的调节, 因此可通过调节阀门特性 来计算主蒸汽流量, 国内外学者对此展开了大量研 究。YONG 和 HALIMI 等 ${ }^{[4-5]}$ 用组合阀代替主汽阀 和控制阀, 通过数值模拟计算并与试验结果对比, 组合阀的流量特性与试验结果吻合良好, 并且降低 了调门损失，其结果可应用于阀门的设计和控制。 GIORGIO $^{[6]}$ 利用 CFD 对调节阀的流量特性进行了 优化和分析，并建立了调节阀的线性化曲线并对调 节阀的特性进行预测，成功应用于一个联合循环电 厂的运行控制。ANDREW 等 ${ }^{[7]}$ 提出一种基于试验数 据预测调门特性系数的方程, 可以应用在各种类型 调门的设计。张宝等 ${ }^{[8]}$ 基于汽轮机流量特性试验, 根据调节阀综合开度, 整定优化机组控制函数, 以 提高机组控制性能。

对于配汽方式的优化方面, 也有许多文献进行 了相关研究 ${ }^{[9-11]}$ 。YIN 等 ${ }^{[12]}$ 建立汽轮机阀门管理的控 制系统的模型, 对阀门控制系统的参数整定方法进 行了定量研究, 得到顺序阀运行的流量特性曲线; 马琳等 ${ }^{[13]}$ 研究顺序阀关闭时调节级的特性参数和 流量特性, 分析重叠度对调节级的影响, 并确定了 最佳重叠度, 提出了调节级效率计算的简化模型, 为机组配汽优化提供了参考; 雷志伟等 ${ }^{[14]}$ 通过模型 自适应算法建立了调门顺序阀下的流量特性模型并 进行优化仿真, 将其应用于某 $660 \mathrm{MW}$ 机组上, 计 算结果表明所建模型改善了调节阀流量特性线性度 并降低调节门重叠度; 李玲等 ${ }^{[15]}$ 提出在对机组进行 各种运行工况下的试验时, 考虑负荷和阀位的影响, 确定各工况下最低热耗值, 进而获得最佳的定滑压 运行曲线。

$300 \mathrm{MW}$ 机组配有 6 个调门, 结构比较复杂, 加之在目前的电力市场中, 该容量机组多为调峰机 组, 常年处于低负荷下运行, 本文以此类型机组为
研究对象，通过变工况计算获得调节阀和调节级的 组合特性曲线，建立理论计算模型。该模型可利用 调节阀门开度和调节级后压力得到准确的调节阀流 量, 并实现了对流量特性的优化, 得到了合理的阀 门重叠度。经过与现场调节阀特性试验数据进行对 比验证, 由模型计算得到的配汽函数能准确反映现 场组合特性。基于此，综合负荷和阀位的影响，获 得机组热耗增量在不同阀位下的变化情况，优化机 组配汽方式并进行分析, 利用机组现场运行数据进 行对比, 验证模型具有可行性, 并对机组运行方式 进一步优化，得到机组最佳运行滑压曲线和运行方 案, 降低了变负荷运行时的热耗, 为现场试验优化 提供了理论基础, 同时也为其他类型机组优化运行 提供指导。

\section{1 调节阀和调节级组合特性建模}

\section{1 调节阀和调节级组合特性计算模型}

调节阀和调节级的物理模型如图 1 所示。

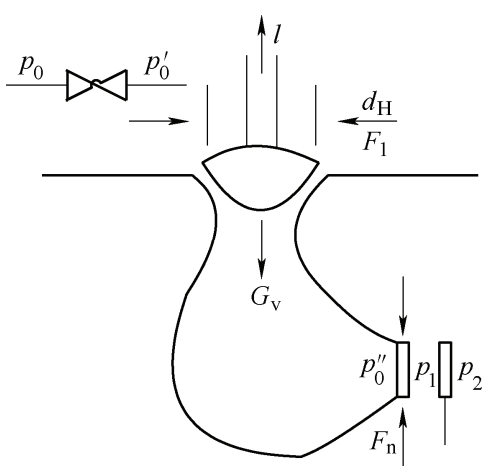

图 1 调节阀与调节级示意图

流过调节阀流量 $G_{1}$ 可表示为 ${ }^{[16]}$

$$
\begin{gathered}
G_{1}=\frac{0.648}{\sqrt{p_{0} v_{0}}} \beta_{1} \chi F_{\mathrm{v}} p_{0}{ }^{\prime} \\
\chi=f\left(\varepsilon_{1}, V\right) \\
\beta_{1}=\sqrt{1-\frac{\left(\varepsilon_{1}-\varepsilon_{\mathrm{cr}}\right)^{2}}{\left(1-\varepsilon_{\mathrm{cr}}\right)^{2}}} \\
\varepsilon_{1}=\frac{p_{0}^{\prime \prime}}{p_{0}^{\prime}}
\end{gathered}
$$

式中, $F_{\mathrm{v}}$ 为调节阀公称通流面积; $p_{0}^{\prime}$ 为调节阀前压 力; $v_{0}$ 为主汽门前比体积, $p_{0}$ 为主汽门前的压力; $\varepsilon_{1}$ 为调节阀前后压比; $\varepsilon_{\mathrm{cr}}$ 为临界压比, 对于过热蒸 汽 $\varepsilon_{\mathrm{cr}}=0.5457$ 。 $\chi$ 为调节阀的相对流量系数, 是调 节阀前后压比 $\varepsilon_{1}$ 和调节阀开度 $V$ 的函数 ${ }^{[16-17]} ; \beta_{1}$ 为 调节阀的彭台门系数，是调节阀压比 $\varepsilon_{1}$ 的函数。

为便于计算, 将 $\chi$ 和 $\beta_{1}$ 组合, 定义 $\beta_{1} \chi$ 为调节 
阀综合流量系数。通过式(2)、(3)可得

$$
\beta_{1} \chi=f\left(\varepsilon_{1}, V\right)
$$

现代汽轮机组很少配备调节阀门后压力测点, 无法直接计算 $\varepsilon_{1}$ 。但汽轮机在运行时可测得调节级 后压力, 因此要对调节级进行分析。

流过调节阀所对应调节级喷嘴组的流量 $G_{2}$ 可 表示为

$$
\begin{gathered}
G_{2}=\frac{0.648}{\sqrt{p_{0} v_{0}}} \mu \beta_{2} F_{\mathrm{n}} p_{0}{ }^{\prime \prime} \\
\beta_{2}=\sqrt{1-\frac{\left(\varepsilon_{2}-\varepsilon_{\mathrm{cr}}\right)^{2}}{\left(1-\varepsilon_{\mathrm{cr}}\right)^{2}}} \\
\varepsilon_{2}=\frac{p_{1}}{p_{0}^{\prime \prime}} \\
\varepsilon_{3}=\frac{p_{2}}{p_{0}^{\prime \prime}}
\end{gathered}
$$

式中, $\mu$ 为喷嘴流量系数; $\beta_{2}$ 为喷嘴彭台门系数; $F_{\mathrm{n}}$ 为喷嘴组通流面积; $p^{\prime \prime}{ }_{0}$ 为喷嘴前压力; $p_{1} 、 p_{2}$ 分别为喷嘴后压力和调节级后压力, $\varepsilon_{2}$ 为喷嘴组 压比。

根据质量守恒方程 $G_{1}=G_{2}$ 将式(1)、(6)代入得

$$
\varepsilon_{1}=\frac{p_{0}^{\prime \prime}}{p_{0}^{\prime}}=\frac{F_{\mathrm{v}}}{F_{\mathrm{n}}} \frac{\beta_{1}}{\beta_{2}} \frac{\chi}{\mu}
$$

式中, $F_{\mathrm{v}}$ 与 $F_{\mathrm{n}}$ 的比值对确定机组来说为定值; $\beta_{1}$ 和 $\beta_{2}$ 分别为调节阀压比 $\varepsilon_{1}$ 和喷嘴压比 $\varepsilon_{2}$ 的函数; $\chi$ 是调节阀前后压比 $\varepsilon_{1}$ 和调节阀开度 $V$ 的函数; $\mu$ 为 常数, 取 $\mu=0.97$ 。

令 $\varepsilon_{\mathrm{g}}=\frac{p_{2}}{p_{0}{ }^{\prime}}, \varepsilon_{\mathrm{g}}$ 为调节阀和调节级组合的压比。

根据文献 [16]理论分析, $\varepsilon_{2}$ 和 $\varepsilon_{3}$ 相对应, $\varepsilon_{3}=\varepsilon_{\mathrm{g}} / \varepsilon_{1}$, 调节阀前后压比 $\varepsilon_{1}$ 与调节阀和调节级组 合压比 $\varepsilon_{\mathrm{g}}$ 存在一一对应关系, 式(10)可化为

$$
\varepsilon_{1}=f\left(\varepsilon_{\mathrm{g}}, V\right)
$$

式(11)中调节阀前后压比 $\varepsilon_{1}$ 与 $\varepsilon_{g}$ 以及调节阀开 度 $V$ 之间的函数关系, 称为调节阀与调节级的组合 流量特性。

为实现调节阀门主汽流量的精确计算, 以调节 阀和调节级为研究对象, 建立组合模型, 根据汽轮 机调节阀门热力设计数据和结构数据, 基于 APROS 平台构建仿真模型, 通过变工况热力计算可得到公 式(12)所示对象机组的调节阀和调节级组合流量特 性曲线如图 2 所示, 和调节阀综合流量系数 $\beta_{1} \chi$ 曲 线如图 3。

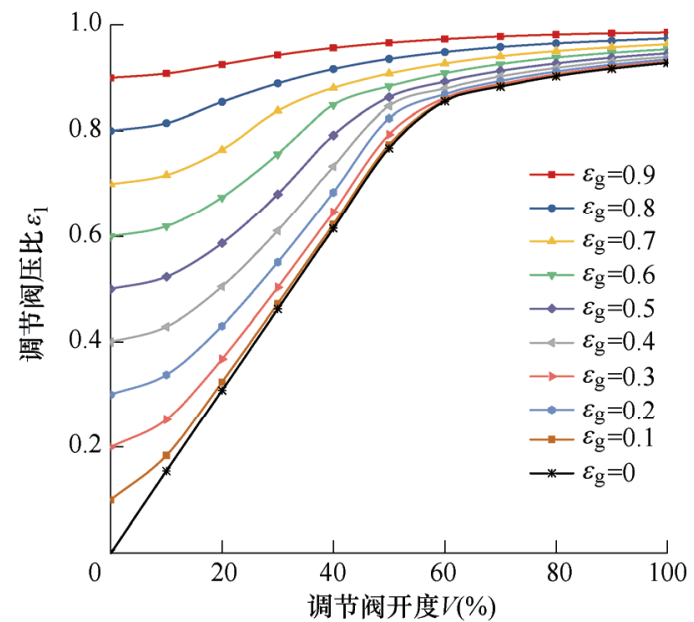

图 2 调节阀和调节级组合流量特性理论曲线

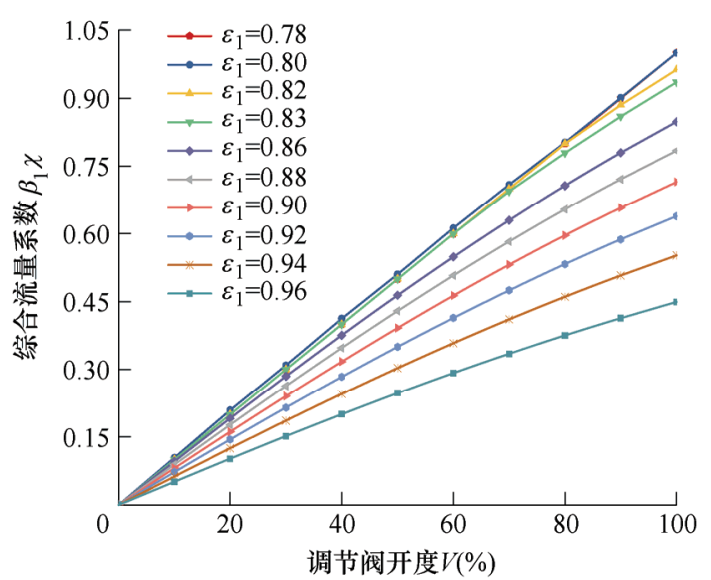

图 3 调节阀综合流量系数曲线

在额定工况下的调节级后压力和通过调节级的 总流量都已知的情况下, 基于图 2 和图 3 可以计算 任意开度下各调节阀的主蒸汽流量以及通过调节级 的总流量，具体计算步骤如下。

（1）设定各调节阀开度 $V$, 并考虑主汽阀额定压 力损失, 假设调节级后压力初值 $p_{20}$, 计算调节阀前 的压力得到组合压比 $\varepsilon_{\mathrm{g}}$ 。

（2）根据调节阀开度 $V$ 和组合压比 $\varepsilon_{\mathrm{g}}$, 由调节阀 和调节级组合流量特性理论曲线可得到调节阀前后 压比 $\varepsilon_{1}$, 并计算调节阀的彭台门系数 $\beta_{1}$ 。

（3）根据式(1)计算每个调节阀的流量以及调节 阀的总流量。

(4) 根据弗留格尔公式计算调节级后压力 $p_{21}$, 将计算值和初值进行比较, 若误差在合理范围内, 则计算完毕; 若不是, 则将计算值返回步骤(1), 直 到误差满足终止条件。

整个迭代计算的流程图如图 4 所示。

\section{2 计算模型的验证}

在调节阀和调节级计算模型中, 调节阀的相对 流量系数 $\chi$ 是关键参数, 对机组的流量特性曲线计 算的准确性具有重要意义。文中计算模型取 $\varepsilon_{\mathrm{g}}=$ 


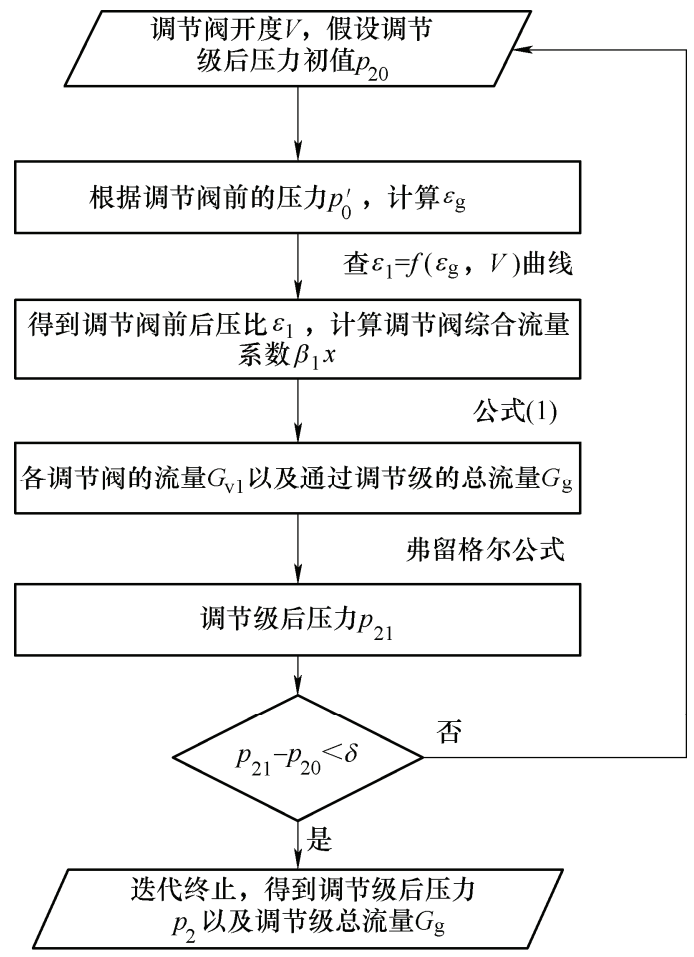

图 4 调节阀与调节级组合特性计算模型流程图

0.4 0.9 范围内, 不同调节阀开度情况下, 调节阀 相对流量系数 $\chi$ 随调节阀前后压比的数据变化与汽 机调节阀试验台的运行数据进行对比验证。

计算模型中调节阀的开度 $V$ 是调节阀的实际行 程 $L$ 与调节阀的总行程 $L_{0}$ 的比值, 而图中试验台的 数据给定的是调节阀实际行程 $L$ 与调节阀公称直径 $D$ 的比值, 调节阀开度与调节阀行程比所对应的关 系为

$$
V=\left(\frac{L}{D}\right) D / L_{0}
$$

图 5 中虚线连接的空心点为文献[5]中提供的调 节阀升程比 $L / D$ 为 $0.1 、 0.09 、 0.07 、 0.06 、 0.05$ 和 0.04 时调节阀相对流量系数随调节阀前后压比的变 化曲线, 实线连接点为理论计算得到的数据。比较 模型计算数据与试验数据的变化趋势可知, 在工作 范围内，计算值与试验值相对流量系数变化趋势一 致, 吻合较好, 模型能较好地反映调节阀的特性。 试验的条件与模型理论计算不尽相同, 文献[5]中以 空气为工质进行试验, 且阀门结构参数并未公开; 计算模型以 $300 \mathrm{MW}$ 调节阀为对象, 以过热蒸汽为 工质进行计算, 所以试验数据与计算值存在一定偏 差。需要注意的是试验台数据与理论计算数据在相 同调节阀开度情况下调节阀前后压比的变化范围不 同, 由于计算时模型取值范围为 $\varepsilon_{\mathrm{g}}=0.4 \sim 0.9$ (图 2), 其在调节阀开度一定的情况下, 调节阀前后压比变 化亦在一定的范围内, 故与试验台前后压比数据的 取值范围不同，对模型的准确性验证无影响。

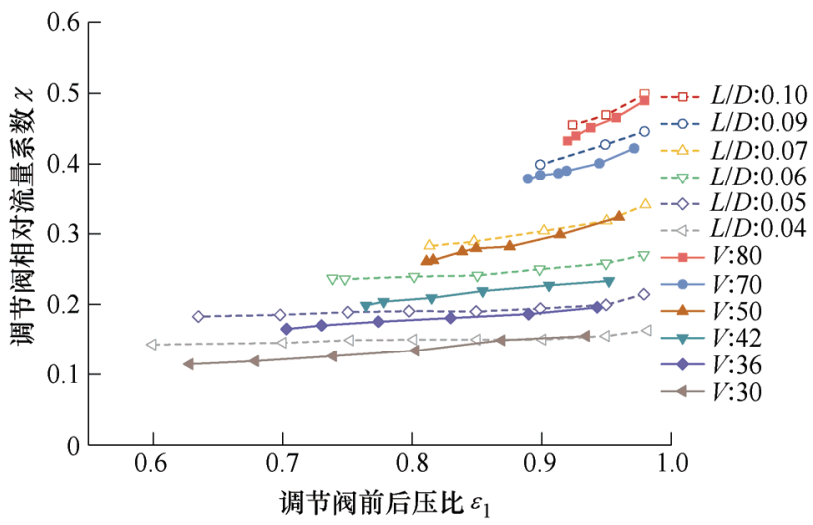

图 5 调节阀相对流量系数对比图

\section{2 计算实例}

\section{1 机组配置}

以某厂 8 号发电机组 $\mathrm{N} 300-16.7 / 537 / 537$ 型汽轮 机为例进行变工况计算。该汽轮机配有两个高压主 汽门、6 个高压调节阀和对应的 6 组喷嘴。自动主 汽门和调节阀的排列顺序如图 6 所示。

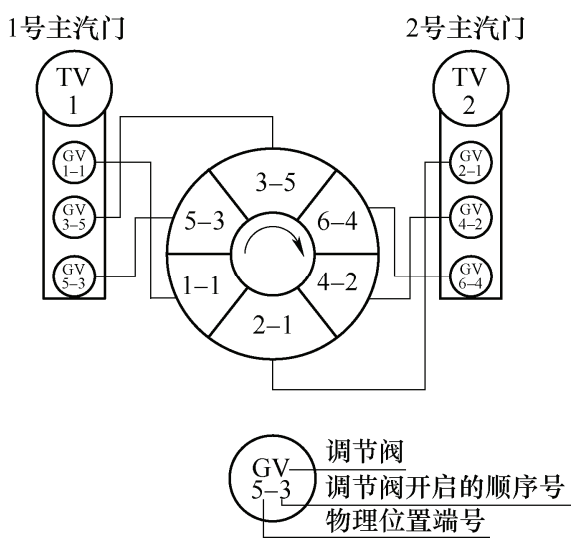

图 6 机组原阀门配置和开启顺序

在已有工作的基础上 ${ }^{[18-20]}$, 在额定进汽参数下, 基于上述调节阀和调节级组合特性计算模型，不考 虑重叠度, 进行变工况定压计算, 得到机组顺序阀 流量特性以及各调节阀的特性曲线(调节阀开启顺 序 GV6+3 $\rightarrow \mathrm{GV} 5 \rightarrow \mathrm{GV} 4 \rightarrow \mathrm{GV} 1 \rightarrow \mathrm{GV} 2$ )如图 7 所示。

由图 7 分析可知，在不设置重叠度情况下，随 着阀门顺序开启，顺序阀流量特性曲线呈非线性变 化, 汽轮机控制系统调节阀门开度时, 会产生流量 波动。

\section{2 调节阀和调节级组合模型应用}

根据调节阀和调节级组合特性理论数学模型, 基于 APROS 平台 ${ }^{[21-23]}$, 对喷嘴配汽汽轮机调节阀 和调节级建模，根据实际机组 DEH 设置的重叠度， 进行计算, 得到对象机组的调节阀和调节级组合流 量特性，并与通过现场试验获得的整定后的配汽函 数进行对比验证, 如图 8 所示。 


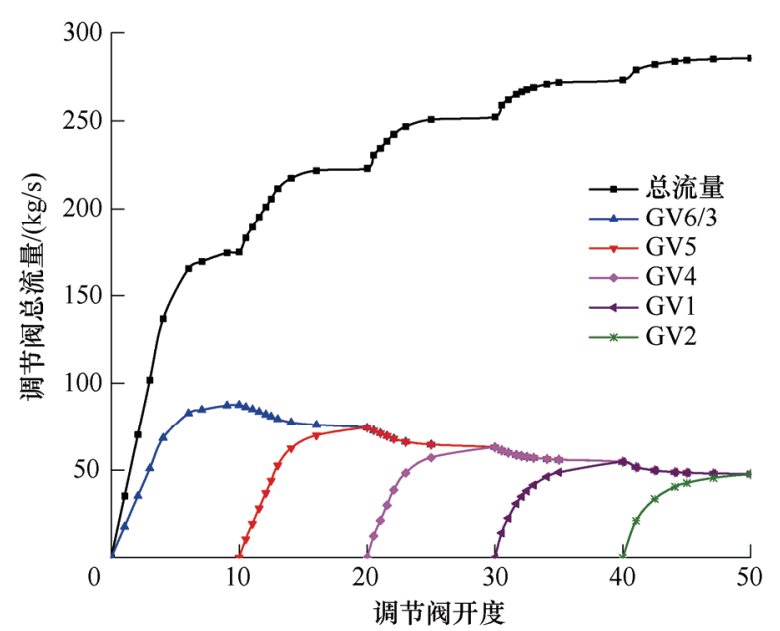

图 7 调节阀联合流量特性曲线

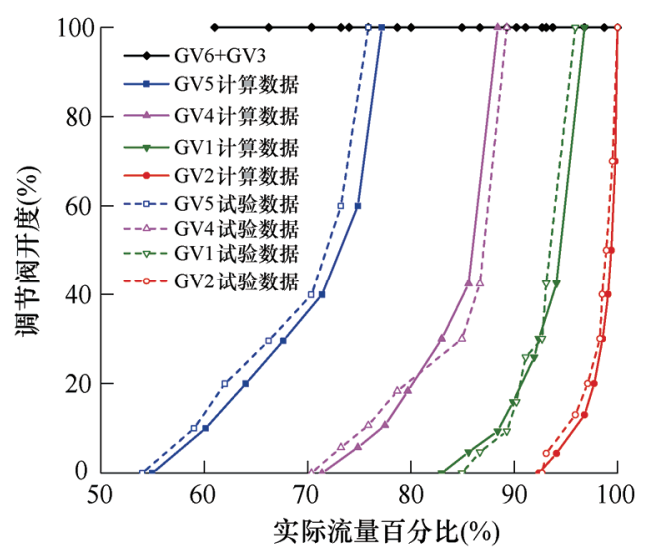

图 8 机组配汽曲线比较图

由图 8 中数据的对比验证可知, 模型计算的调 节阀和调节级组合流量特性与现场试验数据变化趋 势相吻合, 机组的配汽曲线具有一致性, 能够很好 的反映实际运行工况。现场试验时机组受运行因素 影响, 存在测量误差, 导致试验数据与模型计算数 据有一定的偏置, 流量比例的误差在 $2 \%$ 以内。

由图 8 现场试验得到的机组配汽函数可知, 机 组实际运行时设置的重叠度还有优化的可能。为实 现优化, 以阀门控制函数为对象, 根据模型组合 流量特性, 将顺序阀流量特性表示为与流量指令 的关系。

设 $C$ 为机组流量指令, 一般 $C=[0,100]$, 顺序阀 流量则是任意指令下各阀门的流量和。

$$
G(C)=\sum_{i=1}^{n} F\left(\varepsilon_{\mathrm{g}}, H_{i}(C)\right)
$$

式中, $V=H_{i}(C)$ 为第 $i$ 个阀门的控制函数, $F\left(\varepsilon_{\mathrm{g}}, V_{i}\right)$ 是各调节级与调节阀组合的流量特性, 是 压比 $\varepsilon_{\mathrm{g}}$ 和调节阀开度 $V$ 的函数。

设阀门之间的重叠度为 $\delta_{i}$, 重叠度反映在指令 区间上为 $\varphi_{i}=H_{i}^{-1}\left(1-\delta_{i}\right)$ 。为保证流量指令和实际流 量呈线性关系, 将流量指令与实际流量相对应, 进
行反演迭代计算，计算得到新的控制函数及阀门 间的重叠度。得到优化后的调节阀特性曲线如图 9 所示。

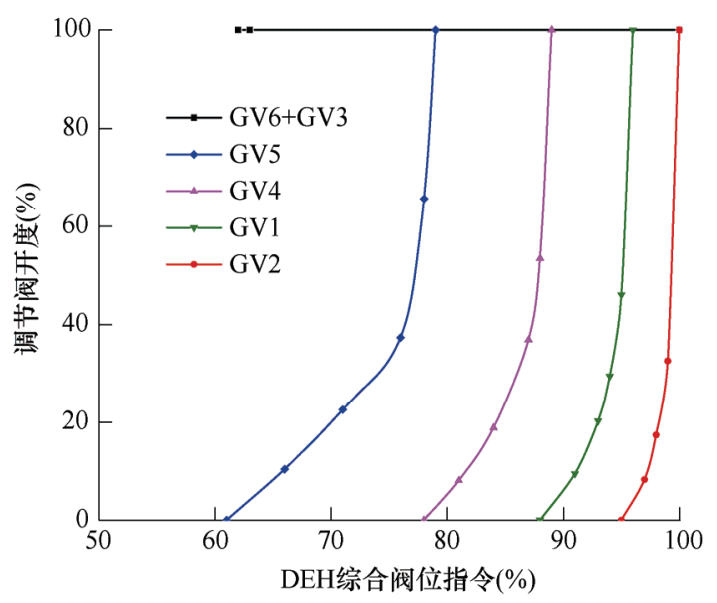

图 9 优化后调节阀特性曲线

优化后流量指令与蒸汽流量之间具有良好的 线性关系，通过计算，每一个阀门开启时，前阀的 压比均在 $0.85 \sim 0.9$ 。根据压力重叠度定义，各阀门 压力重叠度在合理的范围内。根据图 9 可以看到, 相较于机组原配汽曲线，优化后的特性调节阀之间 的重叠度更加合理，提高了流量特性的线性度和可 控性。

将优化后的各调节阀开度指令(对应 DEH 综合 阀位指令)与实际流量特性数据进行处理, 得到曲线 如图 10 所示。

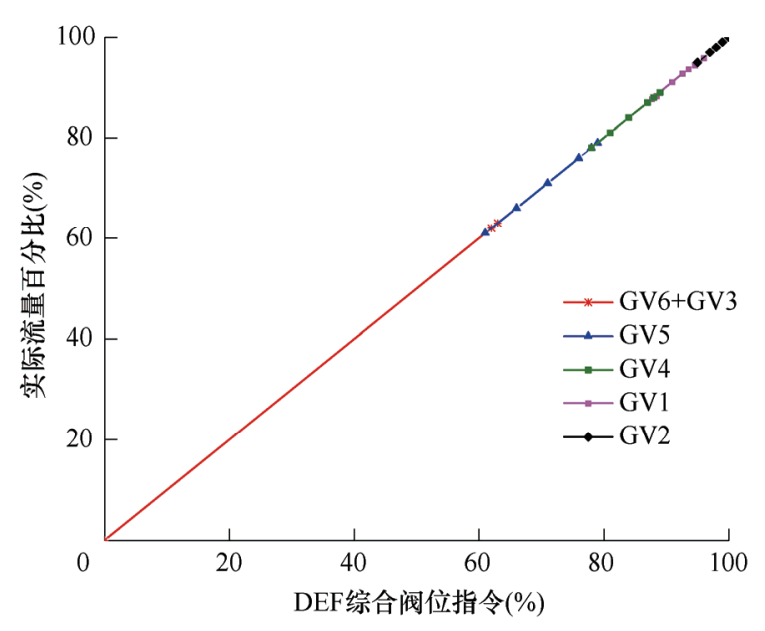

图 $10 \mathrm{DEH}$ 综合阀位指令与实际流量关系曲线

由图 10 可以看出, 对配汽函数进行优化后, 其 $\mathrm{DEH}$ 综合阀位指令与实际流量得到了良好的线性 度，该配汽方式下模型计算的调节阀流量特性与理 想的调节阀流量特性基本吻合, 不会产生 DEH 阀位 指令变化很小时，调节阀开度变化巨大的现象，而 造成实际流量变化剧烈, 影响模型的准确性。 


\section{3 配汽方式优化}

\section{1 机组配汽优化计算}

汽轮机组配汽优化是指通过试验, 得到不同配 汽方式下机组在不同功率负荷下的效率，选取合理 的运行主汽参数和配汽方式, 保证汽轮机组在变负 荷运行时, 能够得到最高的运行效率, 并达到功率 负荷的要求。

在阀门流量特性得到优化, 阀门重叠度更加合 理的基础上, 进行配汽优化试验。基于 APROS 平 台, 对某厂 8 号机组进行变工况运行优化分析。选 取五个典型负荷点, 在各负荷下, 联合负荷和阀位, 改变阀门开度, 来调节主汽压力, 保持定功率运行, 计算得到各负荷点不同配汽方式下的热耗增量, 得 到不同配汽方式下机组热耗率增量的变化曲线如图 11 所示。

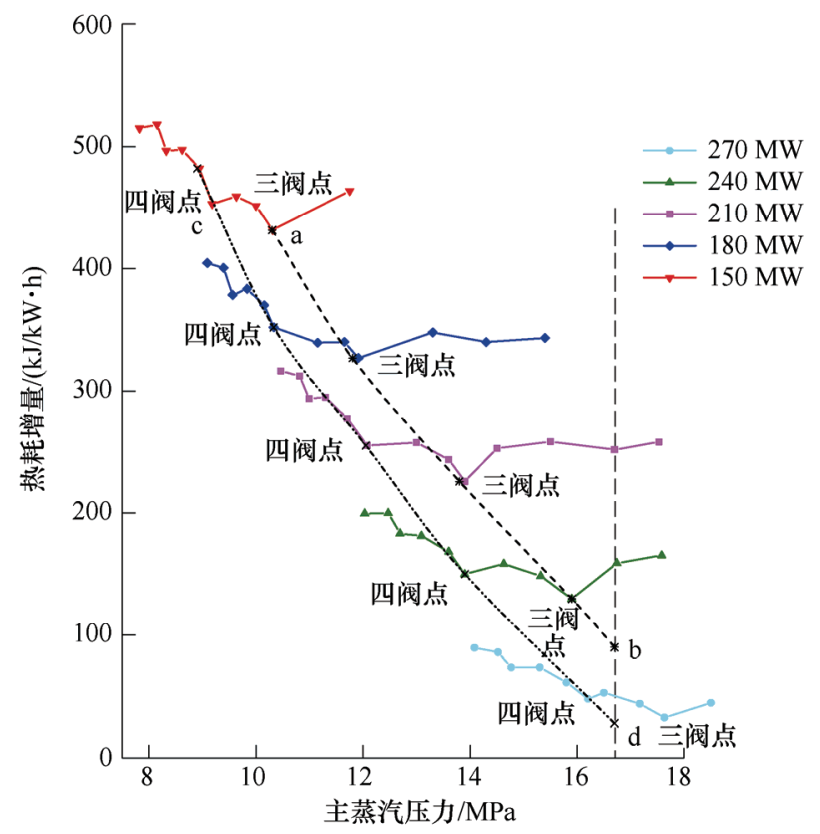

图 11 机组变压运行热耗与主汽压力关系曲线

由图 11 可知, 主蒸汽压力随阀位指令变化, 热 耗在同一负荷下随阀位和主汽压力变化而波动, 由 于阀门节流损失在各阀点处最小, 热耗波动规律呈 “正弦” 变化, 波动范围随阀点个数减少而增大。

\section{2 配汽优化试验验证}

在得到机组变压运行热耗率增量的变化曲线以 及滑压曲线(图 11)后, 将其与某厂 8 号机组实际运 行的试验数据进行对比, 对模型的准确性进行验证。

某厂 8 号机组在实际运行时, 采用 4 阀点滑压定压的配汽方式, 其各调节阀之间存在一定的重叠 度。现场试验数据显示, 在前四阀全开时, 阀门 GV1 存在约为 $7 \%$ 的开度, 如图 11 中虚线 $\mathrm{cd}$ 所示。由于
该阀门开度很小, 流量比较小, 阀门产生的节流效 应不会造成很大影响，机组可看作在近 4 阀点滑压 运行。为使配汽方案与现场工况基本一致, 在机组 背压 $5.39 \mathrm{kPa}$ 下, 模型通过 DEH 综合阀位指令将机 组各调节阀设定在相同的阀位进行滑压运行，获得 了机组运行的初压值, 将其与现场实际运行时的初 压曲线进行对比，如图 12 所示。

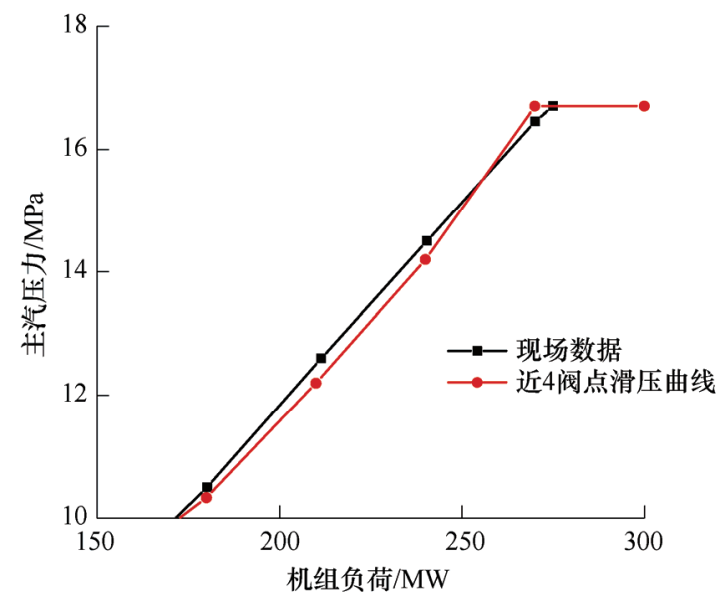

图 12 机组原滑压曲线、模型计算滑压曲线比较

由图 12 可知, 在背压和配汽方式等与现场实际 运行条件基本一致的情况下，经过模型计算得到的 滑压曲线与现场实际运行的初压曲线相比较, 主汽 压力偏差较小, 变化趋势吻合, 两者一致性较好。 因此模型能较好反映机组的实际运行工况。试验数 据与模型计算存在偏差，是因为试验时机组受时间 条件限值, 所选运行工况点有限, 选取的工况不一 定是最优点。根据模型可计算运行范围内任意工况 点, 从中进行寻优, 得到的是最优运行工况。

\section{3 配汽优化深度分析}

通过验证模型能准确反映机组在现场的实际运 行情况，在此基础上，对机组的配汽方式展开寻优。 由图 11 进行分析, 机组实际运行方式的经济性不是 最佳。经过计算得到, 在图 11 中选取的各负荷下, 机组在约 $250 \mathrm{MW}$ 负荷下采用 3 阀点滑压运行，相 比于 4 阀点滑压方式, 其热耗更低, 经济性更好。

在图中连接各工况最佳运行点即热耗率增量 的最低点, 如图 11 虚线 $a b$ 所示, 得到机组主蒸汽 压力随负荷变化曲线如图 13 所示, 即机组最优初 压曲线。

由图 13 中可以得到, 原厂滑压曲线与其他曲线 存在很大的差异。这是因为机组在运行时受到各种 因素的影响, 使得机组运行特性偏离设计值。由图 13 可知, 原滑压曲线在低负荷工况下, 仍然保持着 相对较高的主汽压力, 这使得给水泵耗功变大; 在 依照该滑压曲线运行时, 需要减小调节阀的开度来 


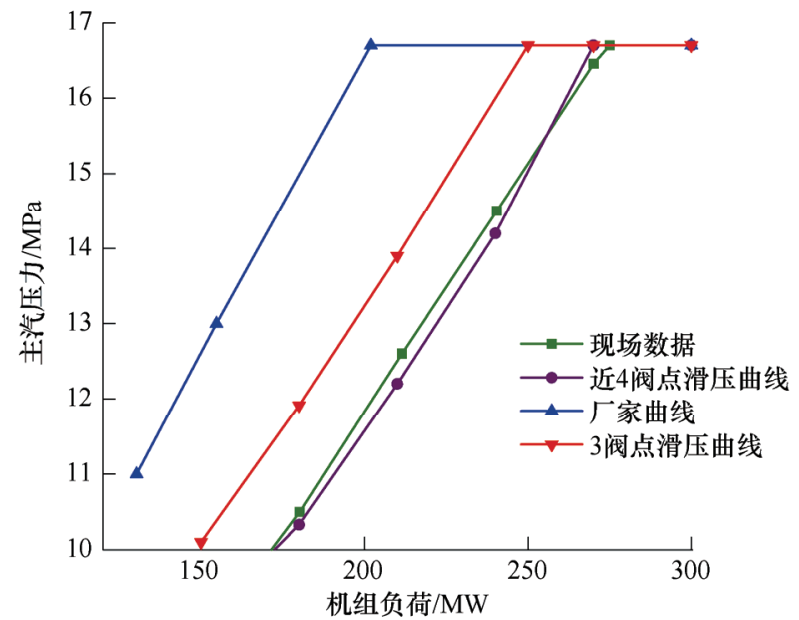

图 13 机组最优初压曲线

维持较高的主汽压力, 这会使节流损失增大, 从而 降低了机组运行的经济性。因此, 按照此滑压曲线 运行, 机组经济性较差。

综上, 由此得到的该机组最优配汽方式如表 1 所示。当运行负荷在 $250 \mathrm{MW}$ 以下时, 采取 3 阀点 滑压运行; 负荷在 $250 \sim 300 \mathrm{MW}$ 时, 机组保持主 汽压力定压运行。

表 1 最优配汽方式

\begin{tabular}{cccc}
\hline 负荷 $/ \mathrm{MW}$ & 主汽压力 $/ \mathrm{MPa}$ & 运行方式 & 阀门开启情况 \\
\hline $250 \sim 300$ & 16.7 & 定压 & 只有 1 阀节流 \\
$150 \sim 250$ & $10.2 \sim 16.7$ & 3 阀点滑压 & 3 阀全开 \\
\hline
\end{tabular}

根据表 1 给出的最优运行方式, 在额定主汽压 力 $16.7 \mathrm{MPa}$ 下, 3 阀点对应负荷以下的工况运行时, 三个阀门全开, 阀门节流损失小, 提高了汽轮机效 率, 同时给水泵的耗电会减少; 在高于 3 阀点对应 的负荷运行时, 保持主汽压力不变, 三个阀门全开, 无节流损失，第 4 个阀门部分开启，有节流损失。 存在节流损失的调节阀个数减少, 机组循环热效率 得到了提高。

\section{4 结论}

(1) 针对喷嘴配汽汽轮机提出调节阀和调节级 组合特性计算模型, 通过公开文献的试验数据验证, 证明该模型具有较高的精度, 能够准确的反映调节 阀流量特性。根据组合压比和开度即可得到准确的 调节阀和调节级流量。

（2）基于 APROS 平台, 利用计算模型对调节级 和调节阀进行修正, 模型可以很好的反映现场实际 情况。计算得到的调节阀流量特性与现场试验数据 吻合较好。

（3）组合特性模型可以应用于阀门流量特性优
化。根据计算得到的顺序阀流量特性, 以控制函数 为目标进行优化, 流量特性线性度得到改善, 阀门 间重叠度更加合理，提高了阀门的可控性。

(4) 根据计算得到的机组各工况下热耗增量随 阀位和主汽压力变化曲线, 机组在 3 阀点滑压运行 时热耗更低, 节流损失更小, 以此得到的最优滑压 曲线运行经济性优于原滑压运行曲线, 该优化方案 降低了机组低负荷运行时的热耗。优化结论是在机 组运行范围内广泛寻优的基础上提出的, 弥补了现 场难以进行大量试验的不足, 为现场试验寻优提供 了理论基础。

\section{参 考 文 献}

[1] 中国电力企业联合会. 2017-2018 年度全国电力供需形 势分析预测报告[R/OL]. [2017-02-01]. http: //www.cec. org.cn/guihuayutongji/gongzuodongtai/2018-02-2018/177 584.html.

China Electric Power Enterprise Federation. The analysis and forecast report of 2017-2018 national power supply and demand situation [R/OL]. [2017-02-01]. http: //www. cec.org.cn/guihuayutongji/Gongzuodongtai/2018-02-2018/ 177584.html.

[2] 国家发展改革委员会, 国家能源局. 能源发展“十三五” 规划[R]. 北京: 人民出版社, 2016 .

The National Development and Reform Commission and The National Energy Administration. The 13th five-year plan for energy development of the people's republic of china[R]. Beijing: Chinese People's Publishing House, 2016.

[3] KUBIK M L, COKER P J, BARLOW J F. Increasing thermal plant flexibility in a high renewables power system[J]. Applied Energy, 2015，154: 102-111.

[4] YONG H Y, KUNE Y S. Engineering analysis of mass flow rate for turbine system control and design[J]. Nuclear Engineering and Design, 2011, 241: 4061-4078.

[5] HALIMI B, KIM S H, KUNE Y S. Engineering of combined valve flow for power conversion system[J]. Energy Conversion and Management, 2013, 65: 448-455.

[6] GIORGIO Z. Experimental and numerical investigation into the aerodynamics of a novel steam turbine valve and its field application[J]. Journal of Engineering for Gas Turbines and Power. 2014, 136: 1-11.

[7] ANDREW G, FRAWLEY P. Experimental parametric equation for the prediction of valve coefficient $\left(\mathrm{C}_{\mathrm{v}}\right)$ for choke valve trims[J]. International Journal of Pressure Vessels and Piping, 2011, 88(2-3): 109-118.

[8] 张宝, 㚞印龙, 顾正皓, 等. 大型汽轮机流量特性试验 [J]. 发电设备, 2012，26(2): 73-76. 
ZHANG Bao, FAN Yinlong, GU Zhenghao, et al. Flow characteristic test for large steam turbines[J]. Power Equipment, 2012, 26(2): 73-76.

[9] PETER B, PHILIPP V, BALKOWSKI I, et al. A new emergency stop and control valves design : Part 1 - experimental verification with scaled models[C]// ASME Turbo Expo 2014: Turbine Technical Conference and Exposition. 2014: V01BT27A001.

[10] LIESE E. Modeling of a steam turbine including partial arc admission for use in a process simulation software environment[C]// ASME 2011 International Mechanical Engineering Congress and Exposition. 2014: 429-437.

[11] YANG Xinshe, HOSSEINI S, GANDOMI A H. Firefly algorithm for solving non-convex economic dispatch problems with valve loading effect[J]. Applied Soft Computing Journal, 2012, 12(3): 1180-1186.

[12] YIN Changjie, LIU Jizhen. Study on valve management of DEH for steam turbine[J]. Energy \& Power Engineering, 2013, 5(4): 319-323.

[13] 马琳, 胥建群, 曹祖庆. 基于量纲分析的汽轮机调节级 变工况特性研究 $[\mathrm{J}]$. 中国电机工程学报, 2014, 34(5): 778-783.

MA Lin, XU Jianqun, CAO Zuqing. Research on varying condition characteristic of steam turbine governing stage based on dimensional analysis[J]. Proceedings of the Chinese Society for Electrical Engineering, 2014, 34(5): 778-783.

[14] 雷志伟, 张兴, 陈涛, 等. 基于模型自适应汽轮机调节 门流量特性优化研究及应用[J]. 中国电力, 2018, 51(3): 21-28.

LEI Zhiwei, ZHANG Xing, CHEN Tao, et al. Research of steam turbine governing valve flow characteristic optimization based on adaptive model[J]. Electric Power, 2018, 51(3): 21-28.

[15] 李玲, 胥建群, 石永锋. $600 \mathrm{MW}$ 汽轮机组运行方式优 化研究 [J]. 发电设备, 2010，24(6): 396-400.

LI Ling, XU Jianqun, SHI Yongfeng. Operation mode optimization for a $600 \mathrm{MW}$ steam turbine unit[J]. Power
Equipment, 2010, 24(6): 396-400.

[16] 曹祖庆. 汽轮机变工况特性[M]. 北京: 水利水电出版 社, 1991.

CAO Zuqing. Varying condition characteristic of steam turbine[M]. Beijing: Water Resources and Electric Power Press, 1991.

[17] 胥建群, 曹祖庆. 汽轮机调速系统和调节级数学模型 [J]. 汽轮机技术，1991，31(1): 15-21.

$\mathrm{XU}$ Jianqun, CAO Zuqing. Turbine speed governor system and the mathematical model of governing stage[J]. Turbine Technology, 1991, (1): 15-21.

[18] MA Lin, XU Jianqun, et al. The influence of overlap degree research on nozzle governing characteristic[J]. Energy and Power Engineering, 2013， 5: 642-645.

[19] 马琳, 胥建群. $600 \mathrm{MW}$ 汽轮机喷嘴配汽方式下阀门重 叠度影响的研究 $[\mathrm{J}]$. 发电设备, 2013, 27(6): 382-385. MA Lin, XU Jianqun. Study on influence of valve overlapping degree of $600 \mathrm{MW}$ nozzle governing steam turbines[J]. Power Equipment, 2013， 27(6): 382-385.

[20] XU Jianqun, MA Lin, SUN Youyuan, et al. Research on characteristics of varying conditions for nozzle governing stage based on dimensional analysis[J]. Energy, 2014, 65: 590-595.

[21] PUSKA E. APROS couplings from core to containment[R]. Finland : VTT Technical Research Centre, 2005.

[22] HANNINEN M. Phenomenological extensions to APROS six-equation model[R]. Finland: VTT Technical Research Centre, 2009.

[23] LAAKSO P. Methods of simulation assisted automation testing[R]. Finland: VTT Technical Research Centre, 2005.

作者简介: 张浩峰, 男, 1992 年出生。主要研究方向为火电机组性能分 析与优化。

E-mail: 245565723@qq.com

胥建群(通信作者), 男, 1959 年出生, 博士, 教授。主要研究方向为汽 轮机运行特性与理论建模、热力透平数值模拟等。

E-mail: qlj1062@163.com 